\title{
El desarrollo de los componentes del model de prevención para sujetos no obligados: de acuerdo a la Ley 30424
}

\begin{abstract}
Jhuliana Atahuamán Páucar
Abogada por la Universidad Nacional Mayor de San Marcos. Máster en Ciencias Jurídicas por la Universitat Pompeu Fabra de Barcelona. Máster en Compliance por Barcelona School of Management. Estudios de Doctorado por Barcelona School of Management.
\end{abstract}

SUMARIO:

I. Introducción.

II. Sujetos obligados.

III. Sistema de la Ley 34024 —-modificada por el Decreto Legislativo 1352 y por la Ley 30835-.

1. Encargado de prevención.

2. Identificación, evaluación y mitigación de riesgos.

3. Implementación de procedimientos de Denuncia.

4. Difusión y Capacitación periódica del Modelo de Prevención.

5. Evaluación y monitoreo continuo del Modelo de Prevención.

* El artículo fue recibido con fecha 04-09-18. 


\title{
RESUMEN:
}

Este artículo analiza la Ley 30424, Ley que regula la responsabilidad administrativa de las personas jurídicas, incluyendo los sujetos obligados y no obligados por la ley, los elementos que la componen y los modelos de prevención de delitos. Para desarrollarlos, el autor presenta instrumentos legales nacionales e internacionales como la Norma Técnica Peruana Oficializada NTP-ISO 37001:2017 sobre sistemas de gestión antisoborno, el Programa Anticorrupción de Ética y Cumplimiento para las Empresas emitido por la Oficina de las Naciones Unidas contra la droga y el delito y el proyecto de reglamento de la Ley 30424.

Palabras clave: Responsabilidad Administrativa, Personas Jurídicas, Modelo de Prevención de Delitos, Antisoborno, Anticorrupción, Delitos.

\begin{abstract}
:
The following article addresses Law 304214, the Law that regulates the administrative responsibility of legal entities, including the obligated and the non-obligated subjects by the law, its components and crime prevention models. In order to explain them, the author presents national and international legal instruments such as the Peruvian Technical Regulation NTP-ISO 37001:2017 regarding anti-bribery management systems, the Anti-corruption of Ethics and Compliance Program for Companies issued by the United Nations Office against drugs and crime and the Law 304214 regulation bill.

Keywords: Administrative Responsibility, Legal Entities, Crime Prevention Model, Anti-bribery, Anti-corruption, Crime.
\end{abstract}

\section{INTRODUCCIÓN}

Cuando una empresa en el Perú desea implementar un modelo de prevención de delitos muchas veces no sabe qué es lo que necesita y qué es lo que más se ajusta a sus intereses y necesidades. Seguramente ha escuchado en la prensa que ha entrado en vigencia una ley que regula modelos de prevención de delitos para las personas jurídicas, pero no entiende en qué consisten, cómo se hacen, cuánto tiempo tomará elaborarlos e implementarlos, cuánto le costará diseñarlos, etc. Frente a esto lo que probablemente hará es consultar a su abogado penalista de cabecera para que le explique en qué consisten esos modelos de prevención y qué es el denominado Compliance.

Antes de optar cualquier modelo, se tendrá que responder a la siguiente pregunta: ¿la organización es sujeto obligado de informar a la Unidad de Inteligencia Financiera del Perú en adelante, "UIF-Perú" - la existencia de operaciones sospechosas - de lavado de activos o financiamiento de terrorismo- o no? La ley señala taxativamente qué empresas tienen esa obligación en el Perú; y que por lo tanto tendrán que adoptar el llamado Sistema de Prevención de Lavado de Activos y Financiamiento de terrorismo-SPLAFT. Para todas las demás organizaciones - que no son considerados sujetos obligados - y que por tanto no tienen la obligación de informar operaciones sospechosas a la UIF-Perú podrán implementar facultativamente un modelo de prevención de delitos de acuerdo a la Ley 30424 - modificada por el Decreto Legislativo 1352 y por la Ley 30835-.

Es preciso señalar que ningún modelo es mejor ni peor que el otro, ni previene mejor la comisión de delitos que el otro. Cada sistema obedece a necesidades diferentes y está orientado a giros de negocio diferentes, y eso se ve reflejado en los componentes que cada sistema exige tener. En esta oportunidad desarrollaremos cada uno de los componentes del modelo de prevención basado en la Ley 30424, modificada dos veces.

Otro instrumento legal que emplearemos para complementar la descripción del modelo de la Ley 30424 es la Norma Técnica Peruana Oficializada NTP-ISO 37001:2017-Sistemas de gestión antisoborno, -en adelante, "NTP-ISO 37001:2017"-; la cual es una adopción de la versión en inglés de la Norma Internacional ISO 37001:2016 —Anti-bribery Management Systems - Requirements with guidance for use- - Esta norma fue preparada por el Instituto Nacional de Calidad-INACAL y está disponible a través de su página web. 
Otro instrumento legal es el Programa Anticorrupción de Ética y Cumplimiento para las Empresas: Guía práctica, emitido por la Oficina de las Naciones Unidas contra la droga y el delito en el 2013 —en adelante, el "Programa"—. Este documento es una guía práctica que muestra las medidas que las empresas pueden tomar para establecer un programa anticorrupción de ética y cumplimiento efectivo. Es interesante el enfoque con el que se desarrolla esta guía pues luego de la exposición de las recomendaciones que el modelo propone, se muestra un práctico cuadro sintetizando todos los requisitos antes expuestos $y$, además, a diferencia de muchos instrumentos legales, elabora requisitos especiales para pequeñas empresas. Es importante señalar, que ninguno de estos dos instrumentos es de obligatorio cumplimiento; sin embargo, ayudan a complementar aquellos aspectos que quizás la norma peruana no tenga ampliamente desarrollados.

Finalmente, la Ley 30424 tiene un Proyecto de Reglamento' el cual desarrolla con mayor amplitud cada uno de los 5 componentes que la Ley exige. Aunque aún no ha entrado en vigencia, lo emplearemos de guía para complementar el significado de cada ítem.

Este trabajo pretende proporcionar al lector lego en la materia una mirada inicial destinada a clarificar el panorama lleno de incertidumbres y preguntas propio de una sociedad poco familiarizada con el cumplimiento de la ley.

\section{SUJETOS OBLIGADOS}

Para decidir qué modelo de prevención se debe diseñar y luego implementar, lo primero que debe hacerse es diferenciar a los llamados sujetos obligados de aquellos que no lo son. Sujeto obligado es aquella persona natural o jurídica que se dedique a cualquiera de las actividades abajo mencionadas. Es la Ley quien coloca la obligación/responsabilidad sobre los hombros de ciertos sujetos pues considera que la actividad que realizan resulta especialmente atractiva - dada su funcionalidadpara poder lavar dinero sucio y/o financiar el terrorismo a través de aquella. Estas organizaciones, sin proponérselo siquiera, podrían facilitar el lavado de activos proveniente de actividades ilícitas, es por eso que tienen que poner más atención que el resto de organizaciones para que la entidad encargada pueda detectar alguna actividad sospechosa.

Ahora bien, recordemos que el ordenamiento jurídico peruano ha sido bastante cambiante. A lo largo de los años, desde la creación de la UIF-Perú a través de la Ley $27693^{2}$, se han ido aumentando la cantidad de sujetos obligados. A la fecha, con las idas y venidas legislativas, tenemos en el Perú a los siguientes sujetos obligados: i) las empresas del sistema financiero y del sistema de seguros y las demás comprendidas en los artículos 16 y 17 de la Ley General del Sistema Financiero y del Sistema de Seguros y Orgánica de la Superintendencia de Banca y Seguros, y los corredores de seguros, ii) las empresas emisoras de tarjetas de crédito, iii) las cooperativas de ahorro y crédito, iv) las empresas que venden y compran divisas, v) las empresas que se dedican al servicio postal de remesa y/o giro postal, vi) las empresas que se dedican al préstamo y/o empeño, vii) los administradores de bienes, empresas y consorcios, viii) las sociedades agentes de bolsa, de productos e inter-

1. Documento que a la fecha de cierre de este artículo aún no entraba en vigencia y cuya disposición de publicación en la página web del Ministerio de Justicia se autorizó mediante Resolución Nº061-2018-JUS de fecha 27 de febrero de 2018.

2. Ley 27693, Artículo 1 - Objeto de la Unidad de Inteligencia Financiera del Perú: "Créase la Unidad de Inteligencia Financiera del Perú, con personería jurídica de derecho público, con autonomía funcional, técnica y administrativa, encargada de recibir, analizar, tratar, evaluar y transmitir información para la detección del lavado de activos y/o del financiamiento del terrorismo; así como de coadyuvar a la implementación por parte de los sujetos obligados del sistema para detectar operaciones sospechosas de lavado de activos y/o del financiamiento de terrorismo (...)". 
mediarias de valores, ix) las sociedades mutuos, fondos de inversión y fondos colectivos, $\mathrm{x}$ ) la Bolsa de Valores, otros mecanismos centralizados de negociación e instituciones de compensación y liquidación de valores, xi) la Bolsa de Productos, xii) las empresas que se dedican a la compra y venta de vehículos, embarcaciones y aeronaves, xiii) las empresas constructoras $y / 0$ inmobiliarias, xiv) los agentes inmobiliarios, xv) las empresas que se dedican a la explotación de juegos de casinos y/o máquinas tragamonedas, y/o juegos a distancia utilizando el internet o cualquiera otro medio de comunicación, xvi) las empresas que se dedican a la explotación de apuestas deportivas a distancia utilizando el internet o cualquier otro medio de comunicación, xvii) las empresas que se dedican a los juegos de lotería y similares, xviii) los hipódromos y sus agencias, xix) los agentes de aduanas, $x x$ ) los notarios, xxi) las empresas mineras, xxii) las empresas que se dedican al comercio de joyas, metales y piedras preciosas, monedas, objetos de arte y sellos postales, xxiii) los laboratorios y empresas que producen y comercializan insumos químicos y bienes fiscalizados, xxiv) las empresas que distribuyen, transportan y/o comercializan insumos químicos que pueden ser utilizados en la minería ilegal, bajo control y fiscalización de la Superintendencia Nacional de Aduanas y de Administración TributariaSUNAT, xxv) las empresas que se dedican a la comercialización de las maquinarias y equipos que se encuentran comprendidos en las Subpartidas nacionales $\mathrm{N}^{\circ} 84.29, \mathrm{~N}^{\circ} 85.02$ y N 87.01 de la Clasificación Arancelaria Nacional, xxvi) las empresas que se dedican a la compraventa o importaciones de armas y municiones, xxvii) las empresas que se dedican a la fabricación o comercialización de materiales explosivos, xxviii) las empresas que se dedican a la financiación colectiva o participativa y que operan a través de plataformas virtuales y xxix) los abogados y contadores públicos colegiados, que de manera independiente o en sociedad, realizan o se disponen a realizar en nombre de un tercero o por cuenta de este, de manera habitual, las siguientes actividades: a) compraventa de bienes inmuebles, b) administración del dinero, valores, cuentas del sistema financiero $\mathrm{u}$ otros activos, c) organizar aportaciones para la creación, operación o administración de personas jurídicas y d) compraventa de acciones o participaciones sociales de personas jurídicas. Si la organización no se encuentra en la lista mencionada, le corresponde — si lo considera conveniente- implementar el modelo de prevención que la Ley 30424 describe.

\section{SISTEMA DE LA LEY 34024 -MODIFICADA POR EL DECRETO LEGISLATIVO 1352 Y POR LA LEY 30835-}

¿En qué consiste el sistema de la Ley 30424, Ley que regula la responsabilidad administrativa de las personas jurídicas?

La norma mencionada sufrió una modificación a través del Decreto Legislativo 1352 publicada en el Diario Oficial El Peruano el 7 de enero de 2017. Esta modificación afectó a los artículos 1 , $2,3,4,5,6,7,11,12,13,14,16,17$ y 18 de la Ley 30424; y con la última modificatoria a través de la Ley 30835 del 11 de julio de 2018, se modificaron los artículos 1, 9 y 10 de la última versión de la Ley 30424.

La norma precisa qué entiende por personas jurídicas, ellas serán las entidades de derecho privado, asociaciones, fundaciones, organizaciones no gubernamentales, comités no inscritos, sociedades irregulares, entes que administran un patrimonio autónomo, las empresas del Estado peruano o sociedades de economía mixta. Como se aprecia, la norma ha tratado de tener un concepto amplio que abarque la mayoría de formas organizacionales.

El gran incentivo para que las personas jurídicas opten por implementar este modelo de prevención es la eximente de responsabilidad por la comisión de los delitos de cohecho, cohecho activo transnacional, lavado de activos, financiamiento del terrorismo, tráfico de influencias y colusión - simple y agravada- Sin embargo, se debe precisar que la eximente no se obtiene automáticamente por la sola presencia de un modelo de prevención, éste deberá contemplar los componentes que la ley reclama y además estar debidamente implementados y ser eficientes. 
Este modelo de prevención debe contener 5 apartados necesariamente. Estos son: a) encargado de prevención, b) canal de denuncias, c) procedimientos de denuncia, d) difusión y capacitación periódica y e) evaluación y monitoreo.

Los componentes que la norma señala son los siguientes:

\section{Encargado de prevención.}

Ni la Ley 30424 ni sus modificatorias definen qué es un encargado de prevención. Lo que resalta es una característica: la autonomía de sus funciones; por su parte el Proyecto de Reglamento de la Ley 30424, a través del artículo 34 además de la autonomía, recalca la independencia y autoridad. Asimismo, señala como funciones las de velar por la aplicación, ejecución, cumplimiento y mejora continua del modelo de prevención, y señala un tema también importante el cual es la asignación de recursos que le permitan a este encargado de prevención un adecuado funcionamiento operativo. Como se observa, ambos instrumentos legales se complementan y hacen más completas las funciones y atribuciones del encargado de prevención.

La NTP-ISO 37001 en su sección A. $6^{3}$ dota de contenido a los requerimientos que hemos señalado líneas arriba y considera además otras atribuciones para el encargado de prevención. Por ejemplo, cuando se menciona independencia, de acuerdo a la NTP-ISO 37001, esto significa que el encargado de prevención en la medida de lo posible no esté involucrado personalmente en las actividades de la organización que esté expuesto al soborno - tranquilamente puede ampliarse a los delitos de lavado de activos y financiamiento de terrorismo, tráfico de influencias y colusión-. La medida que se sugiere para poder cumplir esto es designar a una persona que a tiempo completo - exclusividad- realice las labores de encargado de prevención. Ahora bien, en una organización pequeña o mediana, probablemente no esté dentro del presupuesto pagar a una persona que únicamente se dedique a la labor de cumplimiento, por lo que tendrá que asignar a un colaborador que ya labore dentro de la organización para que además de sus labores profesionales deba cumplir con el rol de asegurador de cumplimiento. Diferente será la situación en una organización de proporciones más grandes, en donde sí cuente con los recursos suficientes y además sea necesario que una persona - o todo un departamento- se encargue del control de cumplimiento. La NTP-ISO:37001 menciona también la autoridad, en este apartado menciona que el encargado de prevención deberá recibir de parte de la alta dirección de la organización poderes suficientes para poder realizar con eficacia sus responsabilidades. Las características que no aparecen en la Ley 30424 ni en el Proyecto del Reglamento son la competencia y la posición. La primera de ellas se refiere a los requisitos del encargado de prevención, se recomienda que éste cuente con educación, formación o experiencia apropiada y la capacidad personal para afrontar las exigencias que la función amerite. Si bien esto puede resultar obvio, no es menos cierto que la realidad demuestra que muchas organizaciones optarán por designar a un colaborador que ya se encuentre laborando dentro de la organización y por lo general será alguien del departamento jurídico o recursos humanos, pues erróneamente se piensa que dichas áreas son similares al área de cumplimiento. Otra característica es la posición que tenga el encargado de prevención dentro del organigrama de la organización. Esta posición debe ser adecuada de tal manera que le permita escuchar y respetar sus opiniones. Finalmente, un aspecto que tampoco menciona la Ley 30424 ni el Proyecto de Reglamento y que sí lo hace la NTP-ISO:37001 es el acceso directo de reporte del encargado de prevención a la alta dirección. La razón es evitar que la información que se reporte llegue incompleta y/o inoportunamente a la alta dirección de la 
organización. Probablemente de no hacerlo así el encargado de prevención tenga que reportar frente a otro gerente y/o director directamente, con la finalidad que la información no llegue defectuosa ni a destiempo.

\section{Identificación, evaluación y mitigación de riesgos.}

La identificación de riesgo plasmado a través de un mapa de riesgos es el reflejo de la situación de la organización a quien se le implementará el modelo de prevención. En dicho análisis, ya que estamos dentro del ámbito de la Ley 30424 lo que se hará es poner énfasis principalmente en los delitos que la ley cubre como importantes: cohecho activo, cohecho activo transnacional, lavado de activos, financiamiento de terrorismo, tráfico de influencias y colusión. Será a criterio de la propia organización si es que la evaluación de riesgos se extienda también a otros delitos que estén fuera del alcance de la ley, y ello no será incompatible con la implementación del modelo.

La evaluación de riesgos se realiza en base a un completo análisis de los procesos que contiene y desarrolla la organización. Naturalmente todos los procesos - actividades, productos y/o servicios- no estarán vinculados a riesgos que se materialicen en comisión o posible comisión de ilícitos o violación del Código de Conducta, es por ello que en el mapa de riesgos solo contendrá aquellos procesos que importe controlar y posteriormente mitigar.

En este punto ni la Ley 30424 ni el Proyecto de Reglamento abordan el cómo del desarrollo de la evaluación de riesgos, quien sí lo hace es la NTP-ISO:37001. Como la misma norma señala aquella puede ser utilizada tanto en organizaciones pequeñas, medianas y grandes.
Aunque esta norma técnica fue pensada para prevenir, detectar y enfrentar el delito de soborno, bien la metodología que expone puede ser una guía útil para los demás delitos que la Ley 30424 abarca.

La metodología que se use puede variar. Por ejemplo, la NTP-ISO:37001 propone un ejemplo de cómo la organización puede abordar la evaluación de riesgos 4 . Partiendo de la idea que cada organización es diferente y única - dependiendo de su giro de negocio, tamaño, complejidad, número de procesos, número de personal, si cotiza o no en bolsa, si pertenece o no a una multinacional, etc.-, cada organización dará prioridad a aquellos riesgos identificados e implementará los controles necesarios respectivos, quedará su criterio también la ponderación de los riesgos identificados — bajo, medio o alto o también en una escala de 5 a 7 criterios o incluso puede ser aún más detallado-así como el nivel de tolerancia hacia ellos ${ }^{5}$. En cuanto al tamaño de la organización, si es una de tamaño pequeño bastará con una gestión de controles centralizados en manos de pocas personas, mientras que una organización más grande -en donde los riesgos surjan de varios lados- necesitará contar con controles apoyados en un grupo más grande. Otro aspecto es el lugar y sector en donde opera la organización opera o espera operar. La NTP-ISO:37001 señala que contar con un mapeo geográfico en donde se especifique la incidencia ${ }^{6}$ de casos de soborno ayuda a controlar a la organización y poner más o menos alarmas para su prevención. Sin embargo, esta metodología puede aplicarse también para el resto de delitos. En cuanto a los procesos con los que cuente la organización se pondrá atención a su naturaleza, magnitud y complejidad, tanto por parte de sus propios colaboradores, como de clientes, futuros clientes, proveedores, agentes, intermediarios, socios comerciales, etc.

4. Norma Técnica Peruana-ISO 37001, pág. 44 y ss.

5. También conocido con la definición de apetito al riesgo.

6. Los indicadores pueden ser el número de denuncias, investigaciones sentencias ocurridas en una determinada jurisdicción fiscal y/o judicial. 


\section{Implementación de procedimientos de denuncia.}

Nuevamente ni la Ley 30424 modificada ni el Proyecto de Reglamento dotan de contenido al procedimiento de denuncia. Otra vez recurriremos a la NTP-ISO 37001 para conocer qué elementos contendrá la política de procedimiento de denuncia. En primer lugar, aquí deben diferenciarse por un lado la política de denuncias en sí; y por otro los medios que conforman el canal de denuncias - protocolo de denuncias-. En el primer apartado me refiero al documento en donde se exponga el objetivo, los sujetos, el alcance, el medio, conductas infractoras, confidencialidad, sanciones, prohibición de represalias, protección al denunciante, entre otros. En el segundo apartado, me refiero a los medios ofrecidos por la organización a los usuarios de poder reportar una denuncia, comúnmente son una línea telefónica, un correo electrónico, una página web, denuncia directa a superior jerárquico, recursos humanos o encargado de prevención.

La NTP-ISO:37001 lo regula como planteamiento de inquietudes ${ }^{7}$ y coincide con varios puntos señalados líneas arriba. Señala que debe existir un fomento para que las personas reporten de buena fe alguna conducta o posible conducta que vaya contra el Código de Conducta, alguna política específica o la ley. La confidencialidad en cuanto al reporte de la denuncia -entiéndase sobre la identidad del denunciante, de otras personas involucradas - y además de la misma investigación que la denuncia de origen. Aunque este último aspecto no es mencionado por la NTP-ISO:37001, es necesario que exista un comité que se encargue de llevar a cabo el desarrollo de las investigaciones y que estas tengan también carácter confidencial -lo cual es diferente a secretismo-. Otro punto es la denuncia anónima, si bien animarse a reportar un hecho con revestimiento delictuoso puede ser difícil, la admisión de denuncias anónimas resulta de gran ayuda para que así no se pierda la oportunidad de conocer ningún reporte de denuncias. Esto a su vez lleva a otro componente: el otorgamiento de garantías tanto para el denunciante como para el denunciado. Para el primero, se plasmará a través de la prohibición expresa de represalias y para el segundo a través de llevar a cabo la investigación respetando sus derechos y garantizando la presunción de inocencia hasta desvirtuarla con un resultado desfavorable. A través del canal de denuncias, además, la organización podrá tomar conocimiento de varios aspectos, uno de ellos será el tipo de infracción con más incidencia de comisión, las áreas más vulnerables a cometer una infracción, si tiene eficacia o no el modelo implementado y con toda esa información podrá actualizar el mapa de riesgos e incidir en los puntos que hagan falta.

\section{Difusión y capacitación periódica del modelo de prevención.}

Se pueden tener las mejores políticas elaboradas dentro del modelo de prevención, pero si estas no son comunicadas y publicitadas a todos los destinatarios adecuada y eficazmente pueden caer en saco roto y no llegar a nada. Es por ello que el modelo de prevención exige a través de este apartado que exista un compromiso de parte de la organización para difundir todas las políticas que tienen y no solo eso, explicar el funcionamiento de otro componente, esto es, del canal de denuncias.

Como se aprecia, todos los componentes del modelo de prevención están relacionados entre sí y tienen una razón de ser para estar dentro del modelo. Este cuarto componente exige que se publicite la importancia del modelo de prevención, las razones por las cuales se ha decidido implementarlo en la organización, quién o quiénes son los que se encuentran a cargo de ello poniendo especial énfasis en la explicación del canal de denuncia.

La Ley 30424 no indica los temas a abordar, pero sí lo hace el Proyecto de Reglamento, estos son: a) política de cumplimiento - y su procedimien- 
to-y prevención de delitos, b) riesgos de incurrir en los delitos que contempla el modelo y sus respectivas consecuencias tanta para la organización como para el colaborador infractor, c) circunstancias en las que puede presentarse alguna situación que implique la comisión de alguno de los delitos referidos en base a las funciones que tenga el grupo destinatario de la capacitación, d) formas de reconocimiento y enfrentamiento de las situaciones de riesgo, e) identificación de los canales de denuncia y su respectivo procedimiento, $\mathrm{f}$ ) formas de colaboración para la prevención de riesgos y para la mejora del modelo de prevención, g) consecuencias legales del incumplimiento del modelo de prevención e h) información sobre los recursos con los que se cuente para la capacitación.

En cuanto a la NTP-ISO 37001 no ha desarrollado a profundidad este elemento del modelo, como sí lo hace el Programa. Específicamente en el título H. Comunicación y capacitación' ${ }^{9}$, se expone las consideraciones para llevar a cabo actividades de comunicación y capacitación, así como las clases de capacitación que pueden darse.

El Programa enfoca su desarrollo identificando a los destinatarios, es decir elaborar programas de capacitación personalizados grupo por grupo. También se requiere que la alta dirección de la organización muestre su compromiso en el proyecto de implementar el modelo a través de mensajes a todos los colaboradores. Esta guía también lista qué instrumentos y medios a emplear pueden servir para difundir el programa de capacitación. Se aconseja utilizar páginas web, correos electrónicos, boletines, cursos de capacitaciones, etc. El uso de ejemplos que muestren situaciones del día a día de los destinarios es de mucha ayuda, porque así aterrizaran sus problemas objetivos con las normas peruanas y con los valores éticos de la empresa que contempla también el modelo de prevención.
Estas capacitaciones deben quedar registradas y ser resguardadas, aunque ningún instrumento legal señale quien debe ser el garante de este resguardo, se recomiendo que se centralice en el departamento de Compliance o en su defecto por el encargado de prevención ${ }^{10}$. En el registro se recomienda guardar el Programa conteniendo los temas que se impartieron, las fechas y las horas de duración de la capacitación, así como la asistencia de los colaboradores. Se recomienda también hacer evaluaciones luego de completar el ciclo de evaluación a los colaboradores. La calificación debe ser un índice para conocer el grado de conocimiento que los colaboradores tienen acerca del modelo de prevención, se recomienda también resguardar las calificaciones obtenidas de manera confidencial y no ser empleadas para causar perjuicio al colaborador, por el contrario, se deberán tener en cuenta para que se refuercen los temas en un futuro o tener que volver a repetirlos a la brevedad si los resultados salieron desalentadores.

\section{Evaluación y monitoreo continuo del modelo de prevención.}

El último componente corresponde a la evaluación de todo el modelo de prevención. La ley no indica el cómo se debe hacer el monitoreo, sin embargo, el Proyecto de Reglamento sí muestra los mecanismos de retroalimentación que apoyen el mejoramiento permanente. Señala como encargado de este proceso a la alta dirección de la organización - aunque puede contar con el apoyo del encargado de prevención- e indica también que debe resguardarse a través de la documentación debida que así lo demuestre.

La razón de ser de este último componente no es otro que medir los avances y la eficiencia que ha tenido el modelo de prevención en la organización, así como las fallas que puede haber y deben corregirse. De acuerdo al Proyecto los

8. Proyecto de Reglamento de la Ley 30424, artículo 40.

9. Norma Técnica Peruana-ISO 37001, pág. 75.

10. Independientemente de la modalidad que puede ser presencial o virtual. 
aspectos que deben evaluarse son ${ }^{11}:$ a) funcionamiento del modelo de prevención, b) fallas y/o debilidades encontradas, c) detalles de las acciones correctivas realizadas, d) eficacia de las medidas adoptadas para hacer frente a los riesgos identificados y e) oportunidades de mejora del modelo de prevención.

Por su parte, la NTP-ISO $37001^{12}$ recomienda para la evaluación del desempeño señalar los métodos de seguimiento, medición, análisis y evaluación, la fecha - o el cronograma - de evaluaciones, a quien se debe reportar los resultados obtenidos.

Recalca la importancia de conservar la evidencia a través de la documentación en donde consten los resultados obtenidos, el método utilizado y el responsable de ejecutarlo.

Por su lado, el Programa es más profundo y diferencia dos conceptos importantes ${ }^{13}$. El primero es el examen y el segundo la evaluación. Señala que el examen es un estudio en profundidad realizado en un punto del ciclo de vida del modelo de prevención, mientras que la evaluación será el análisis de los resultados obtenidos del examen. Será a partir los criterios de las evaluaciones donde se identificará las necesidades de modificaciones y mejoras. Por qué es útil es esta diferencia, pues porque permitirá al evaluador situarse frente a qué circunstancia se encuentra y tener los indicadores necesarios para plantear las reformas. Una vez realizado ese análisis, este Programa indica que los tipos de modificaciones que pueden darse son aquellos que introduzcan nuevas medidas, que adiciones medidas ya existentes, que reduzcan medidas que hayan resultado ineficientes y/o que se adapten las estrategias planteadas. El Programa explica además qué situaciones deben ser tomadas en cuenta al momento de realizar el examen al modelo. Uno de ellos es la ampliación de la organización a nuevos mercados y operaciones comerciales, la aparición de nuevas relaciones comerciales -entre ellos nuevos proveedores $\mathrm{y} / \mathrm{o}$ clientes-, nuevas estructuras comerciales y con ello la aparición de nuevos procesos - por ejemplo la aparición de una nueva sucursal-, nuevas metas de desempeño, la aparición de nuevos requisitos o normas en la industria donde opera la organización - por ejemplo, como en el caso peruano la ampliación de delitos a través de la modificatoria de la ley-, nuevos requisitos del entorno social o de otras partes interesadas, la aparición de casos de corrupción — también puede ser de lavado de activos, financiamiento de terrorismo, tráfico de influencias y colusión-, los propios resultados de la supervisión interna, los resultados de las auditorías internas y/o externa también muestra una radiografía de cómo se están aplicando las políticas durante los procesos de la organización, la evaluación de los colaboradores - por ejemplo luego de haber recibido el programa de capacitación- y finalmente las referencias con organización dentro de la industria también puede dar perspectiva de lo que se tiene en frente y debe mejorar. Luego de obtener todos los datos de todos los ítems arriba expuestos, corresponderá analizarlos - esto es, evaluarlos-. Los criterios de medición que recomienda el Programa son 3: a) efectividad, b) eficiencia y c) sostenibilidad. Con la primera se busca medir de qué manera las políticas y procedimientos elaborados han contribuido a lograr los objetivos específicos del modelo de prevención; con el segundo se mide la minimización de los costos del modelo de prevención con el tiempo que garantiza los beneficios de las políticas y/o procedimientos; y finalmente con el tercero se hace referencia a los resultados que las políticas y/o procedimientos hayan ayudado a minimizar todos los riesgos identificados.

11. Proyecto de Reglamento de la Ley 30424, artículo 41.

12. Norma Técnica Peruana-ISO 37001, pág. 33.

13. Programa Anticorrupción de Ética y Cumplimiento para las Empresas: Guía práctica, emitido por la Oficina de las Naciones Unidas contra la droga y el delito en el 2013, pág. 105. Nuevamente, aunque el programa se haya elaborado pensando en los delitos de corrupción, nada impide que la metodología queda emplearse como referencia también para aquellos modelos destinados a mitigar riesgos que involucren los delitos de lavado de activos, financiamiento de terrorismo, tráfico de influencias y colusión. 